Research Article

\title{
Distributed Adaptive Output Consensus for High-Order Multiagent Systems with Input Saturation and Uncertain Nonlinear Dynamics
}

\author{
Ming-Can Fan $\mathbb{D}^{1}$ and Wen Qin ${ }^{2}$ \\ ${ }^{1}$ School of Mathematics and Statistics, Huizhou University, Huizhou 516007, Guangdong, China \\ ${ }^{2}$ College of Electrical Engineering and Control Science, Nanjing Tech University, Nanjing 211816, Jiangsu, China
}

Correspondence should be addressed to Ming-Can Fan; mingcan_fan@163.com

Received 16 June 2020; Accepted 21 July 2020; Published 12 August 2020

Guest Editor: Xiao Ling Wang

Copyright (c) 2020 Ming-Can Fan and Wen Qin. This is an open access article distributed under the Creative Commons Attribution License, which permits unrestricted use, distribution, and reproduction in any medium, provided the original work is properly cited.

\begin{abstract}
This paper deals with the leader-following output consensus problem for a class of high-order affine nonlinear strict-feedback multiagent systems with unknown control gains and input saturation under a general directed graph. Nussbaum gain function technique is used to handle the unknown control gains, and the uncertain nonlinear dynamics of each agent is approximated by radial basis function neural networks. Distributed adaptive controllers are designed via the backstepping technique as well as the dynamic surface control approach. It is proved that the closed-loop multiagent systems are semiglobally uniformly ultimately bounded, and the output consensus error can converge to a small region around the origin. Finally, the theoretical results are supported by a numerical simulation.
\end{abstract}

\section{Introduction}

Consensus control of multiagent systems (MAS) has drawn considerable attention in the past two decades due to its broad applications in multiple ground-moving robots [1], unmanned aerial vehicles (UAVs) [2], unmanned surface vessels [3], sensor networks [4], smart grids [5], and synchronization and flocking models [6-8], for instance. The investigation on this topic has been carried out from different perspectives, to mention a few, such as single-integrator or double-integrator $\operatorname{MAS}[9,10]$, general linear MAS [11-13], nonlinear MAS [14-16], fractional-order MAS [17, 18], and high-order MAS $[19,20]$.

Consensus problems of MAS with unknown control gains, including unknown amplitudes of control gains or unknown signs of control gains (the so-called control directions), are gaining researchers' increasing attention in recent years. It is necessary and beneficial to study this topic since the control gains are often unknown in many practical control systems, such as the autopilot design of time-varying ships and unmanned sailboat heading control. There are many results of consensus control for MAS with unknown control gains, for instance, [21-26]. In [21], Nussbaum function-based adaptive control was developed to handle the consensus problem for a firstorder MAS provided that the communication digraph was balanced and weakly connected. By using a Nussbaumlike switching function, the authors in [22] also addressed the first-order consensus problem under a strongly connected communication digraph. In [23], using a novel Nussbaum gain function, consensus for a second-order linearly parameterized MAS was realized under the assumption that the control directions were the same and the communication graph was undirected and connected. Some progress has been made with respect to high-order MAS. In [24], adaptive backstepping-based controllers were designed to guarantee the consensus for high-order MAS under an undirected and connected communication graph. In [25], the authors investigated high-order 
linearly parameterized MAS, while external disturbances were not considered. In [26], high-order MAS with nonlinear dynamics and partially unknown nonidentical time-varying control directions, as well as bounded external disturbances, was considered, where the communication digraph was assumed to be strongly connected. Note that most of the existing works including those mentioned above did not take input saturation into account, which is often encountered in practical applications due to physical limitations of actuators and may cause the instability or damage the control systems' performance [27-31].

Motivated by the limitation of the existing literatures, this paper studies the leader-following output consensus problem for a class of high-order nonlinear MAS subject to input saturation and bounded external disturbances. Moreover, the control gains are assumed to be time varying and unknown for the controller design. This issue has not been mentioned in any existing references to the best of the authors knowledge. Based on the backstepping technique combined with the dynamic surface control (DSC) method, and under the assumption that the communication digraph has a spanning tree, a distributed adaptive neural controller is proposed with the aid of the well-known Nussbaum gain function. It is proved that the closed-loop MAS is semiglobally uniformly ultimately bounded (SUUB), and the output consensus error can converge to a small region around the origin.

Compared with the existing results, the primary contributions of this paper can be summarized as follows. (1) First, the high-order MAS model discussed in this paper is a class of affine nonlinear systems in the strict-feedback form with external disturbances, which is more general than most existing systems regarding output consensus control with unknown control gains [26, 32, 33], where the system dynamics was described with the Brunovsky form or the input saturation was not considered. Hence, the consensus schemes in these references could not be applied. (2) Second, unlike some existing results where the signs of control gains were assumed to be known in advance and the derivative of each control gain function was assumed to be bounded [34] or the unknown nonlinear dynamics satisfied global Lipschitz conditions $[35,36]$, the control gains (including their amplitudes and signs) and the nonlinear dynamics in this paper are completely unknown.

The rest of this paper is organized as follows. In Section 2 , some preliminaries are introduced and the problem of this paper is formulated. Then, a backstepping-based control algorithm is proposed in Section 3, and the closedloop stability is proved in Section 4. In Section 5, a simulation example is given to verify the proposed control algorithm. Finally, some remarks of this paper are concluded in Section 6.

\section{Preliminaries and Problem Statement}

In this paper, a class of high-order affine nonlinear MAS with $N$ followers is considered. The dynamics of each follower is described as follows:

$$
\begin{aligned}
\dot{x}_{i, j} & =h_{i, j}\left(\bar{x}_{i, j}\right)+\ell_{i, j}\left(\bar{x}_{i, j}\right) x_{i, j+1}+d_{i, j}(t), \\
\dot{x}_{i, n_{i}} & =h_{i, n_{i}}\left(\bar{x}_{i, n_{i}}\right)+\ell_{i, n_{i}}\left(\bar{x}_{i, n_{i}}\right) \sigma\left(v_{i}\right)+d_{i, n_{i}}(t), \\
y_{i} & =x_{i, 1}, \quad j=1,2, \ldots, n_{i}-1,
\end{aligned}
$$

where $\bar{x}_{i, j}=\left[x_{i, 1}, x_{i, 2}, \ldots, x_{i, j}\right]^{T} \in \mathbb{R}^{j}, v_{i} \in \mathbb{R}$, and $y_{i} \in \mathbb{R}$ are the state vectors, actual controllers, and outputs of the MAS, respectively, $i=1,2, \ldots, N, d_{i, j}(t)$ are the unknown bounded time-varying external disturbances, the unknown continuous function $h_{i, j}(\cdot)$ represents the uncertain nonlinear dynamics, $\ell_{i, j}(\cdot)$ represents a continuous and unknown time-varying control gain function, and $\sigma(\cdot): \mathbb{R} \longrightarrow \mathbb{R}$ is a standard saturation function defined as $\sigma(x)=\operatorname{sgn}(x) \min \left\{|x|, v^{*}\right\}$, where $v^{*}>0$ is a saturation level constant. The output of the leader is $y_{0}(t)$, which satisfies the following assumption.

Assumption 1. $y_{0}(t), \dot{y}_{0}(t)$, and $\ddot{y}_{0}(t)$ are bounded for $t \geq 0$.

Remark 1. The dynamics of the uncertain MAS (1) is affine nonlinear in the strict-feedback form with external disturbances, which cannot be converted to the Brunovsky form studied in most existing literatures $[26,32,33]$ due to the unknown control gains. Hence, the MAS is more general, and the controller design is more challenging.

Remark 2. The strict-feedback nonlinear MAS (1) may have great potential for practical applications since it can describe many dynamical behaviors, such as robotic systems, flight systems, and biochemical process [37].

In order to use the backstepping technique, the discontinuous saturation nonlinearity $\sigma(\cdot)$ is replaced by a smooth function [38]: $\sigma(x)=v^{*} \times \operatorname{erf}(p x)$, where $p=\sqrt{\pi} /\left(2 v^{*}\right)$ and $\operatorname{erf}(x)=(2 / \sqrt{\pi}) \int_{0}^{x} e^{-t^{2}} \mathrm{~d} t$ is a Gaussian error function, which is a real-valued and continuous differentiable function. Defining $\eta\left(v_{i}\right)=v^{*} \times \operatorname{erf}\left(p v_{i}\right)-q v_{i}$, $q>0$, and then we have $\sigma\left(v_{i}\right)=q v_{i}+\eta\left(v_{i}\right)$.

The purpose of this paper is to present an adaptive neural output consensus controller for MAS (1) such that the semiglobal uniform ultimate boundedness for all the signals in the closed-loop system is ensured, and each follower's output $y_{i}(i=1,2, \ldots, N)$ synchronically tracks the leader's output $y_{0}$. Moreover, the output consensus error can converge to a small region around the origin. To this end, the following assumptions and preliminaries are required.

Assumption 2. There exist constants $\bar{\eta}, q, \bar{q}$, and $\bar{d}_{i, j}$, such that $\left|\eta\left(v_{i}\right)\right| \leq \bar{\eta}, q \in[\underline{q}, \bar{q}]$, and $d_{i, j}(t) \leq \bar{d}_{i, j}$, respectively, $i=$ $1,2, \ldots, N$ and $j=1,2, \ldots, n_{i}$.

Assumption 3. There exist positive constants $\underline{\ell}_{i, j}$ and $\bar{\ell}_{i, j}$ such that the unknown control gains $\ell_{i, j}\left(\bar{x}_{i, j}\right)$ satisfy $\underline{\ell}_{i, j} \leq\left|\ell_{i, j}\left(\bar{x}_{i, j}\right)\right| \leq \bar{\ell}_{i, j}, \forall \bar{x}_{i, j} \in \Omega \subset \mathbb{R}^{j}$.

Definition 1 (see [39]). A continuous function $N(\cdot): \mathbb{R} \longrightarrow \mathbb{R}$ is called a Nussbaum gain function if it satisfies 


$$
\begin{aligned}
& \lim _{s \longrightarrow+\infty} \sup \frac{1}{s} \int_{0}^{s} N(\kappa) \mathrm{d} \kappa=+\infty, \\
& \lim _{s \longrightarrow+\infty} \inf \frac{1}{s} \int_{0}^{s} N(\kappa) \mathrm{d} \kappa=-\infty .
\end{aligned}
$$

In this paper, a Nussbaum gain function $\kappa^{2} \cos ((\pi / 2) \kappa)$ is chosen.

Lemma 1 (see [40]). Let $\Gamma(t)$ and $\kappa_{i}(t)$ be smooth functions defined on $[0, T)$ with $\Gamma(t) \geq 0, \forall t \in[0, T)$, and $N(\cdot)$ be an even smooth Nussbaum gain function. If the following inequality holds:

$$
\Gamma(t) \leq A+e^{-B t} \sum_{i=1}^{N} \int_{0}^{t}\left[\ell_{i}(s) N\left(\kappa_{i}(s)\right)+1\right] \dot{\kappa}_{i}(s) e^{B s} \mathrm{~d} s,
$$

where A represents some suitable constant, $B>0$ is a positive constant, and the value of the time-varying parameter $\ell_{i}(\cdot)$ is located in the unknown intervals $I=\left[l^{-}, l^{+}\right]$with $0 \notin I$, then $\Gamma(t), \kappa_{i}(t)$, and $\sum_{i=1}^{N} \int_{0}^{t}\left[\ell_{i}(s) N\left(\kappa_{i}(s)\right)+1\right] \dot{\kappa}_{i}(s) d s$ are bounded on $[0, T)$.

The communication topology in MAS (1) with $N$ followers is expressed via a digraph $\mathscr{G}=(\mathscr{V}, \mathscr{E}, \mathscr{A})$, where $N$ followers are denoted by a node set $\mathscr{V}=\{1,2, \ldots, N\}$, $\mathscr{E} \subset \mathscr{V} \times \mathscr{V}$ is an edge set, and $\mathscr{A}=\left[a_{i j}\right] \in \mathbb{R}^{N \times N}$ is an adjacency matrix. $a_{i j}>0$ if the $i$-th follower can receive information from the $j$-th follower, otherwise $a_{i j}=0$. Selfloop is not considered, i.e., $a_{i i}=0 . D=\operatorname{diag}\left(d_{1}, d_{2}, \ldots, d_{N}\right)$ is defined as an in-degree matrix with $d_{i}=\sum_{j=1}^{N} a_{i j}$ being the in-degree of the $i$-th follower. The Laplacian matrix of $\mathscr{G}$ is denoted by $\mathscr{L}=D-A=\left[l_{i j}\right] \in \mathbb{R}^{N \times N}$. A digraph contains a spanning tree provided that there exists a directed path from one node (called root node) to every other node in the graph. A leader adjacency matrix $\mathscr{R}=\operatorname{diag}\left(r_{1}, r_{2}, \ldots, r_{N}\right)$ is used to demonstrate the communication between the leader and the follower, where $r_{i}>0$ represents that the leader is a neighbor of the $i$-th follower, and $r_{i}=0$ otherwise.

Assumption 4. The communication digraph $\mathscr{G}$ among the $N$ followers contains a spanning tree, and the leader is a neighbor of the root node.

Since the uncertain nonlinear dynamics of MAS (1) is unknown and continuous, many function approximators can be used, such as neural networks, spline functions, polynomials, and fuzzy systems. Gaussian radial basis function neural network (RBFNN) approach is easy to implement due to its small number of control parameters; therefore, it is widely used in nonlinear function approximation. In this paper, we employ an RBFNN to approximate the unknown function $\Psi(Z): \mathbb{R}^{m} \longrightarrow \mathbb{R}$ on a prescribed compact set $\Omega \subset \mathbb{R}^{m}$, i.e.,

$$
\Psi(Z)=\varrho^{* T} \vartheta(Z)+\omega(Z), \quad Z \in \Omega,
$$

where $Z=\left[z_{1}, z_{2}, \ldots, z_{m}\right] T$ is the input vector, $\varrho^{*}=\left[\varrho_{1}^{*}, \varrho_{2}^{*}, \ldots, \varrho_{s}^{*}\right] T \in \mathbb{R}^{s}$ is the ideal weight vector with $s>1$ being the number of neurons, $\vartheta(Z)=\left[\vartheta_{1}(Z), \vartheta_{2}(Z), \ldots, \vartheta_{s}(Z)\right] T \in \mathbb{R}^{s}$ is the Gaussian basis function vector with $\vartheta_{i}(Z)=\exp \left[-\left\|Z-c_{i}\right\|^{2} /\left(2 w_{i}^{2}\right)\right]$, where $c_{i}$ and $w_{i}$ are the center and the width of the Gaussian function, respectively, $i=1,2, \ldots, s$, and $\varpi(Z)$ denotes the approximation error. It is known that given any positive constant $\varepsilon$, if $s$ is large enough, there exist suitable vectors $\varrho^{*}$ and $\vartheta(Z)$ such that $|\oplus(Z)| \leq \varepsilon$.

\section{Consensus Controller Design}

The distributed adaptive output consensus controller design procedure consists of $n_{i}$ steps using the backstepping technique. To begin with, the following error surfaces for the $j$-th follower are defined:

$$
\begin{aligned}
& e_{i, 1}=\sum_{j=1}^{N} a_{i j}\left(y_{i}-y_{j}\right)+r_{i}\left(y_{i}-y_{0}\right), \\
& e_{i, j}=x_{i, j}-\bar{\gamma}_{i, j}, \quad j=2, \ldots, n_{i},
\end{aligned}
$$

where $\bar{\gamma}_{i, j}$ is the filtered virtual controller, which is acquired through a first-order filter with the virtual controller $\gamma_{i, j}$ as the input. The boundary layer error between $\bar{\gamma}_{i, j}$ and $\gamma_{i, j}$ is defined as $\tilde{\gamma}_{i, j}=\bar{\gamma}_{i, j}-\gamma_{i, j}$.

Step 1. The time derivative of $e_{i, 1}$ in equation (5) is obtained as

$$
\begin{aligned}
\dot{e}_{i, 1}= & \left(r_{i}+d_{i}\right)\left[h_{i, 1}\left(x_{i, 1}\right)+\ell_{i, 1}\left(x_{i, 1}\right) x_{i, 2}+d_{i, 1}\right] \\
& -\sum_{j=1}^{N} a_{i j}\left[h_{j, 1}\left(x_{j, 1}\right)+\ell_{j, 1}\left(x_{j, 1}\right) x_{j, 2}+d_{j, 1}\right]-r_{i} \dot{y}_{0} .
\end{aligned}
$$

Define $\Psi_{i, 1}\left(\zeta_{i, 1}\right):=h_{i, 1}\left(x_{i, 1}\right)-\left(1 /\left(r_{i}+d_{i}\right)\right)\left[\sum_{j=1}^{N} a_{i j}\left[h_{j, 1}\right.\right.$ $\left.\left.\left(x_{j, 1}\right)+\ell_{j, 1}\left(x_{j, 1}\right) x_{j, 2}\right]-r_{i} \dot{y}_{0}\right], \quad \zeta_{i, 1}=\left[x_{i, 1}, x_{j, 1}, x_{j, 2}, \dot{y}_{0}\right]^{T}$. Throughout this paper, let $\widehat{x}$ be the estimates of $x^{*}$, and the corresponding estimation error be $\tilde{x}=\widehat{x}-x^{*}$.

Then, the unknown smooth function $\Psi_{i, 1}\left(\zeta_{i, 1}\right)$ can be approximated by an RBFN as

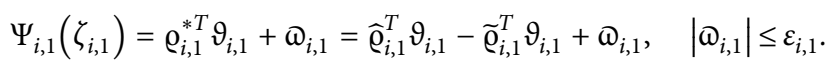

Then, we have

$$
\begin{aligned}
\dot{e}_{i, 1}= & \left(r_{i}+d_{i}\right)\left[\ell_{i, 1}\left(x_{i, 1}\right) x_{i, 2}+\widehat{\varrho}_{i, 1}^{T} \vartheta_{i, 1}-\widetilde{\varrho}_{i, 1}^{T} \vartheta_{i, 1}+\omega_{i, 1}+d_{i, 1}\right] \\
& -\sum_{j=1}^{N} a_{i j} d_{j, 1} .
\end{aligned}
$$

The virtual controllers $\gamma_{i, 2}$ and adaptation laws $\kappa_{i, 1}$ and $\widehat{\varrho}_{i, 1}$ are designed as follows:

$$
\begin{aligned}
& \gamma_{i, 2}=N\left(\kappa_{i, 1}\right)\left(\alpha_{i, 1} e_{i, 1}+\widehat{\varrho}_{i, 1}^{T} \vartheta_{i, 1}\right), \\
& \dot{\kappa}_{i, 1}=\alpha_{i, 1} e_{i, 1}^{2}+e_{i, 1} \widehat{\varrho}_{i, 1}^{T} \vartheta_{i, 1} \\
& \dot{\hat{\varrho}}_{i, 1}=\Lambda_{i, 1}\left(\vartheta_{i, 1} e_{i, 1}-\beta_{i, 1} \widehat{\varrho}_{i, 1}\right),
\end{aligned}
$$


where $\Lambda_{i, 1}$ is a positive-definite matrix, $\alpha_{i, 1}>0$ and $\beta_{i, 1}>0$ are the design parameters. Adjusting the value of $\alpha_{i, 1}$ properly will make the error $e_{i, 1}$ converge to a small neighborhood of the origin. $\beta_{i, 1} \widehat{\varrho}_{i, 1}$ is a modification term with $\beta_{i, 1}$ being a small positive constant, such that $\widehat{\varrho}_{i, 1}$ will not drift to very large values.
Under Assumption 4, we have $r_{i}+d_{i}>0 \forall i \in \mathscr{V}$. Hence, we can construct the Lyapunov function candidate $\Upsilon_{i, 1}=1 /\left[2\left(r_{i}+d_{i}\right)\right] e_{i, 1}^{2}$. Note that $\bar{\gamma}_{i, 2}=\tilde{\gamma}_{i, 2}+\gamma_{i, 2}$, and then we have

$$
\begin{aligned}
& \dot{\Upsilon}_{i, 1}=\frac{1}{r_{i}+d_{i}} e_{i, 1} \dot{e}_{i, 1}=e_{i, 1}\left[\ell_{i, 1}\left(e_{i, 2}+\widetilde{\gamma}_{i, 2}+\gamma_{i, 2}\right)+\widehat{\varrho}_{i, 1}^{T} \vartheta_{i, 1}-\widetilde{\varrho}_{i, 1}^{T} \vartheta_{i, 1}+\varpi_{i, 1}+d_{i, 1}-\frac{1}{r_{i}+d_{i}} \sum_{j=1}^{N} a_{i j} d_{j, 1}\right] \\
& =e_{i, 1}\left[e_{i, 1}\left(e_{i, 2}+\tilde{\gamma}_{i, 2}+N\left(\kappa_{i, 1}\right)\left(\alpha_{i, 1} e_{i, 1}+\widehat{\varrho}_{i, 1}^{T} \vartheta_{i, 1}\right)\right)+\widehat{\varrho}_{i, 1}^{T} \vartheta_{i, 1}-\tilde{\varrho}_{i, 1}^{T} \vartheta_{i, 1}+{\varpi_{i, 1}}+d_{i, 1}-\frac{1}{r_{i}+d_{i}} \sum_{j=1}^{N} a_{i j} d_{j, 1}\right] \\
& =-\alpha_{i, 1} e_{i, 1}^{2}+\left[\ell_{i, 1} N\left(\kappa_{i, 1}\right)+1\right] \dot{\kappa}_{i, 1}+\ell_{i, 1} e_{i, 1} e_{i, 2}+\ell_{i, 1} e_{i, 1} \widetilde{\gamma}_{i, 2}-e_{i, 1} \widetilde{\varrho}_{i, 1}^{T} \vartheta_{i, 1}+e_{i, 1}\left[\varpi_{i, 1}+d_{i, 1}-\frac{1}{r_{i}+d_{i}} \sum_{j=1}^{N} a_{i j} d_{j, 1}\right] \\
& \leq-\alpha_{i, 1} e_{i, 1}^{2}+\left[\ell_{i, 1} N\left(\kappa_{i, 1}\right)+1\right] \dot{\kappa}_{i, 1}+\ell_{i, 1} e_{i, 1} e_{i, 2}+\ell_{i, 1} e_{i, 1} \tilde{\gamma}_{i, 2}+\xi_{i, 1}\left|e_{i, 1}\right|-e_{i, 1} \widetilde{\mathrm{e}}_{i, 1}^{T} \vartheta_{i, 1}
\end{aligned}
$$

where $\xi_{i, 1}=\varepsilon_{i, 1}+\bar{d}_{i, 1}+\left(1 /\left(r_{i}+d_{i}\right)\right) \sum_{j=1}^{N} a_{i j} \bar{d}_{j, 1}>0$. Using Young's inequality [41], the following inequalities hold:

$$
\begin{gathered}
\ell_{i, 1} e_{i, 1} e_{i, 2} \leq e_{i, 1}^{2}+\frac{\ell_{i, 1}^{2} e_{i, 2}^{2}}{4}, \\
\ell_{i, 1} e_{i, 1} \widetilde{\gamma}_{i, 2} \leq e_{i, 1}^{2}+\frac{\ell_{i, 1}^{2} \widetilde{\gamma}_{i, 2}^{2}}{4}, \\
\xi_{i, 1}\left|e_{i, 1}\right| \leq e_{i, 1}^{2}+\frac{\xi_{i, 1}^{2}}{4} .
\end{gathered}
$$

Substituting (14)-(16) into (13), one has

$$
\begin{aligned}
\dot{\Upsilon}_{i, 1} \leq & -\left(\alpha_{i, 1}-3\right) e_{i, 1}^{2}+\left[\ell_{i, 1} N\left(\kappa_{i, 1}\right)+1\right] \dot{\kappa}_{i, 1}+\frac{1}{4} \ell_{i, 1}^{2} e_{i, 2}^{2} \\
& +\frac{1}{4} \ell_{i, 1}^{2} \widetilde{\gamma}_{i, 2}^{2}+\frac{1}{4} \xi_{i, 1}^{2}-e_{i, 1} \widetilde{\varrho}_{i, 1}^{T} \vartheta_{i, 1} .
\end{aligned}
$$

In order to avoid differentiating $\gamma_{i, 2}$, the so-call explosion of the complexity problem is inherent in the backstepping technique, and let $\gamma_{i, 2}$ pass through a first-order filter such that a filtered virtual controller $\bar{\gamma}_{i, 2}$ is acquired:

$$
\begin{aligned}
\tau_{i, 2} \dot{\bar{\gamma}}_{i, 2}+\bar{\gamma}_{i, 2} & =\gamma_{i, 2}, \\
\bar{\gamma}_{i, 2}(0) & =\gamma_{i, 2}(0),
\end{aligned}
$$

where $\tau_{i, 2}>0$ is a small time constant.

A Lyapunov function candidate $\Gamma_{i, 1}(t)$ is given as

$$
\Gamma_{i, 1}(t)=\Upsilon_{i, 1}+\frac{1}{2} \widetilde{\gamma}_{i, 2}^{2}+\frac{1}{2} \widetilde{\varrho}_{i, 1}^{T} \Lambda_{i, 1}^{-1} \widetilde{\varrho}_{i, 1} .
$$

Using (18), we have $\dot{\tilde{\gamma}}_{i, 2}=\dot{\bar{\gamma}}_{i, 2}-\dot{\gamma}_{i, 2}=-\left(\tilde{\gamma}_{i, 2} / \tau_{i, 2}\right)+B_{i, 2}$, where

$B_{i, 2}=-\left(\partial \gamma_{i, 2} / \partial \kappa_{i, 1}\right) \dot{\kappa}_{i, 1}-\left(\partial \gamma_{i, 2} / \partial e_{i, 1}\right) \dot{e}_{i, 1}-\left(\partial \gamma_{i, 2} / \partial \widehat{\varrho}_{i, 1}\right) \dot{\hat{\mathrm{e}}}_{i, 1}$ is a continuous function. Since for any $B_{0}>0$ and $Q>0$, the sets
П: $\left\{\left(y_{0}, \dot{y}_{0}, \ddot{y}_{0}\right): y_{0}^{2}+\dot{y}_{0}^{2}+\ddot{y}_{0}^{2} \leq B_{0}\right\}$ and $\Pi_{i}:=\left\{\sum_{j=1}^{i} 1 /\left[\left(r_{j}+\right.\right.\right.$ $\left.\left.\left.d_{j}\right)\right] e_{j, 1}^{2}+\sum_{j=1}^{i} \gamma_{j, 2}^{2}+\sum_{j=1}^{i} \widetilde{\varrho}_{j, 1}^{T} \Lambda_{j, 1}^{-1} \widetilde{\varrho}_{j, 1} \leq 2 Q\right\}, i=1,2, \ldots, N$, are compact in $R^{3}$ and $R^{3 i}$, respectively, and $\Pi \times \Pi_{i}$ is also compact in $R^{3 i+3}$. Therefore, $\left|B_{i, 2}\right|$ has a maximum $\bar{B}_{i, 2}$ on $\Pi \times \Pi_{i}$, i.e., $\left|B_{i, 2}\right| \leq \bar{B}_{i, 2}$. Similar analysis can be found in [42]. Hence, we have

$$
\tilde{\gamma}_{i, 2} \dot{\tilde{\gamma}}_{i, 2} \leq-\frac{\tilde{\gamma}_{i, 2}^{2}}{\tau_{i, 2}}+\left|\tilde{\gamma}_{i, 2}\right| \bar{B}_{i, 2} \leq-\frac{\widetilde{\gamma}_{i, 2}^{2}}{\tau_{i, 2}}+\widetilde{\gamma}_{i, 2}^{2}+\frac{1}{4} \bar{B}_{i, 2}^{2} .
$$

By taking the time derivative of $\Gamma_{i, 1}$, one has

$$
\begin{aligned}
& \dot{\Gamma}_{i, 1}(t)=\dot{\Upsilon}_{i, 1}+\widetilde{\gamma}_{i, 2} \dot{\tilde{\gamma}}_{i, 2}+\widetilde{\mathrm{\varrho}}_{i, 1}^{T} \Lambda_{i, 1}^{-1} \dot{\tilde{\mathrm{e}}}_{i, 1} \\
& \leq-\left(\alpha_{i, 1}-3\right) e_{i, 1}^{2}+\left[\ell_{i, 1} N\left(\kappa_{i, 1}\right)+1\right] \dot{\kappa}_{i, 1}+\frac{1}{4} \ell_{i, 1}^{2} e_{i, 2}^{2} \\
& +\left(\frac{\bar{\ell}_{i, 1}^{2}}{4}-\frac{1}{\tau_{i, 2}}+1\right) \tilde{\gamma}_{i, 2}^{2}+\frac{1}{4} \omega_{i, 1}^{2}-\beta_{i, 1} \widetilde{\mathrm{e}}_{i, 1}^{T} \widehat{\mathrm{e}}_{i, 1},
\end{aligned}
$$

where $\omega_{i, 1}^{2}=\xi_{i, 1}^{2}+\bar{B}_{i, 2}^{2}$. Note that

$$
\begin{aligned}
-\widetilde{\varrho}_{i, 1}^{T} \widehat{\varrho}_{i, 1} & =-\widetilde{\varrho}_{i, 1}^{T}\left(\widetilde{\varrho}_{i, 1}+\varrho_{i, 1}^{*}\right) \\
& \leq-\left\|\widetilde{\varrho}_{i, 1}\right\|^{2}+\left\|\widetilde{\varrho}_{i, 1}\right\| \cdot\left\|\varrho_{i, 1}^{*}\right\| \\
& \leq-\left(\frac{1}{2}\right)\left\|\widetilde{\varrho}_{i, 1}\right\|^{2}+\left(\frac{1}{2}\right)\left\|\varrho_{i, 1}^{*}\right\|^{2},
\end{aligned}
$$

then we have

$$
\begin{aligned}
\dot{\Gamma}_{i, 1}(t) \leq & -\left(\alpha_{i, 1}-3\right) e_{i, 1}^{2}-\left(\frac{1}{\tau_{i, 2}}-\frac{\bar{\ell}_{i, 1}^{2}}{4}-1\right) \tilde{\gamma}_{i, 2}^{2}-\frac{\beta_{i, 1}}{2}\left\|\widetilde{\varrho}_{i, 1}\right\|^{2} \\
& +\left[\ell_{i, 1} N\left(\kappa_{i, 1}\right)+1\right] \dot{\kappa}_{i, 1}+\frac{\beta_{i, 1}}{2}\left\|\varrho_{i, 1}^{*}\right\|^{2}+\frac{1}{4} \omega_{i, 1}^{2}+\frac{1}{4} \ell_{i, 1}^{2} e_{i, 2}^{2} .
\end{aligned}
$$


Step $j\left(2 \leq j \leq n_{i}-1\right)$. The derivative of the error surface $e_{i, j}=x_{i, j}-\bar{\gamma}_{i, j+1}$ in (6) is obtained as

$$
\dot{e}_{i, j}=h_{i, j}\left(\bar{x}_{i, j}\right)+\ell_{i, j} x_{i, j+1}+d_{i, j}-\dot{\bar{\gamma}}_{i, j+1} .
$$

Define $\Psi_{i, j}\left(\zeta_{i, j}\right):=h_{i, j}\left(\bar{x}_{i, j}\right)-\dot{\bar{\gamma}}_{i, j+1}, \quad \zeta_{i, j}=\left[\bar{x}_{i, j}, \dot{\bar{\gamma}}_{i, j}\right]^{T} \epsilon$ $\mathbb{R}^{j+1}$. This unknown smooth function $\Psi_{i, j}\left(\zeta_{i, j}\right)$ can be approximated by an RBFNN as

$$
\Psi_{i, j}\left(\zeta_{i, j}\right)=\varrho_{i, j}^{* T} \vartheta_{i, 1}+{\varpi_{i, j}}=\widehat{\varrho}_{i, j}^{T} \vartheta_{i, j}-\widetilde{\varrho}_{i, j}^{T} \vartheta_{i, j}+\varpi_{i, j}, \quad\left|\varpi_{i, j}\right| \leq \varepsilon_{i, j} .
$$

The virtual controllers $\gamma_{i, j+1}, j=2, \ldots, n_{i}-1$, and adaptation laws $\kappa_{i, j}$ and $\hat{\varrho}_{i, j}$ are designed as follows:

$$
\begin{aligned}
\gamma_{i, j+1} & =N\left(\kappa_{i, j}\right)\left(\alpha_{i, j} e_{i, j}+\hat{\varrho}_{i, j}^{T} \vartheta_{i, j}\right), \\
\dot{\kappa}_{i, j} & =\alpha_{i, j} e_{i, j}^{2}+e_{i, j} \widehat{\varrho}_{i, j}^{T} \vartheta_{i, j}, \\
\dot{\hat{\varrho}}_{i, j} & =\Lambda_{i, j}\left(\vartheta_{i, j} e_{i, j}-\beta_{i, j} \hat{\varrho}_{i, j}\right),
\end{aligned}
$$

where $\Lambda_{i, j}$ is a positive-definite matrix, $\alpha_{i, j}>0$ and $\beta_{i, j}>0$ are the design parameters.

Applying the Lyapunov function $\Upsilon_{i, j}=(1 / 2) e_{i, j}^{2}$ and noting that $\bar{\gamma}_{i, j+1}=\tilde{\gamma}_{i, j+1}+\gamma_{i, j+1}$, we have

$$
\begin{aligned}
\dot{\Upsilon}_{i, j} & =e_{i, j} \dot{e}_{i, j}=e_{i, j}\left[e_{i, j}\left(e_{i, j+1}+\widetilde{\gamma}_{i, j+1}+\gamma_{i, j+1}\right)+\widehat{\varrho}_{i, j}^{T} \vartheta_{i, j}-\widetilde{\varrho}_{i, j}^{T} \vartheta_{i, j}+\varpi_{i, j}+d_{i, j}\right] \\
& =-\alpha_{i, j} e_{i, j}^{2}+\left[\ell_{i, j} N\left(\kappa_{i, j}\right)+1\right] \dot{\kappa}_{i, j}+\ell_{i, j} e_{i, j} e_{i, j+1}+\ell_{i, j} e_{i, j} \widetilde{\gamma}_{i, j+1}-e_{i, j} \widetilde{\varrho}_{i, 1}^{T} \vartheta_{i, 1}+e_{i, j}\left(\emptyset_{i, j}+d_{i, j}\right) \\
& \leq-\alpha_{i, j} e_{i, j}^{2}+\left[\ell_{i, j} N\left(\kappa_{i, j}\right)+1\right] \dot{\kappa}_{i, j}+\ell_{i, j} e_{i, j} e_{i, j+1}+\ell_{i, j} e_{i, j} \widetilde{\gamma}_{i, j+1}+e_{i, j}\left|\xi_{i, j}\right|-e_{i, j} \widetilde{\varrho}_{i, j}^{T} \vartheta_{i, j},
\end{aligned}
$$

where $\xi_{i, j}={\varpi_{i, j}}_{i}+\bar{d}_{i, j}$. Using Young's inequality, we have

$$
\begin{gathered}
\ell_{i, j} e_{i, j} e_{i, j+1} \leq e_{i, j}^{2}+\ell_{i, j}^{2} \frac{e_{i, j+1}^{2}}{4}, \\
\ell_{i, j} e_{i, j} \widetilde{\gamma}_{i, j+1} \leq e_{i, j}^{2}+\ell_{i, j}^{2} \frac{\widetilde{\gamma}_{i, j+1}^{2}}{4}, \\
e_{i, j}\left|\xi_{i, j}\right| \leq e_{i, j}^{2}+\frac{\xi_{i, j}^{2}}{4} .
\end{gathered}
$$

Substituting the above equations into (29) gives

$$
\begin{aligned}
\dot{\Upsilon}_{i, j} \leq & -\left(\alpha_{i, j}-3\right) e_{i, j}^{2}+\left[\ell_{i, j} N\left(\kappa_{i, j}\right)+1\right] \dot{\kappa}_{i, j}+\frac{1}{4} \ell_{i, j}^{2} e_{i, j+1}^{2} \\
& +\frac{1}{4} \ell_{i, j}^{2} \widetilde{\gamma}_{i, j+1}^{2}+\frac{1}{4} \xi_{i, j}^{2}-e_{i, j} \widetilde{\varrho}_{i, j}^{T} \vartheta_{i, j} .
\end{aligned}
$$

Let $\gamma_{i, j+1}$ pass through a first-order filter with a small time constant $\tau_{i, j+1}>0$ to acquire $\bar{\gamma}_{i, j+1}, j=2, \ldots, n_{i}-1$ :

$$
\begin{aligned}
\tau_{i, j+1} \dot{\bar{\gamma}}_{i, j+1}+\bar{\gamma}_{i, j+1} & =\gamma_{i, j+1}, \\
\bar{\gamma}_{i, j+1}(0) & =\gamma_{i, j+1}(0) .
\end{aligned}
$$

From (32), we have

$$
\dot{\tilde{\gamma}}_{i, j+1}=-\frac{\widetilde{\gamma}_{i, j+1}}{\tau_{i, j+1}}+B_{i, j+1} \leq-\frac{\widetilde{\gamma}_{i, j+1}^{2}}{\tau_{i, j+1}}+\widetilde{\gamma}_{i, j+1}^{2}+\frac{1}{4} \bar{B}_{i, j+1}^{2},
$$

where $B_{i, j+1}=\left(\partial \gamma_{i, j+1} / \partial \kappa_{i, j}\right) \dot{\kappa}_{i, j}+\left(\partial \gamma_{i, j+1} / \partial e_{i, j}\right) \dot{e}_{i, j}+\left(\partial \gamma_{i, j+1} /\right.$ $\left.\partial \widehat{\varrho}_{i, j}\right) \dot{\hat{\varrho}}_{i, j}$ is a continuous and bounded function satisfying $\left|B_{i, j+1}\right| \leq \bar{B}_{i, j+1}$.

Applying the Lyapunov function,

$$
\Gamma_{i, j}(t)=\Upsilon_{i, j}+\frac{1}{2} \widetilde{\gamma}_{i, j+1}^{2}+\frac{1}{2} \widetilde{\varrho}_{i, j}^{T} \Lambda_{i, j}^{-1} \widetilde{\varrho}_{i, j} .
$$

Similar to the previous step, we have

$$
\begin{aligned}
& \dot{\Gamma}_{i, j}(t)=\dot{\Upsilon}_{i, j}+\tilde{\gamma}_{i, j+1} \dot{\tilde{\gamma}}_{i, j+1}+\tilde{\varrho}_{i, j}^{T} \Lambda_{i, j}^{-1} \dot{\tilde{\varrho}}_{i, j} \\
& \leq-\left(\alpha_{i, j}-3\right) e_{i, j}^{2}+\left[\ell_{i, j} N\left(\kappa_{i, j}\right)+1\right] \dot{\kappa}_{i, j}+\frac{1}{4} \ell_{i, j}^{2} e_{i, j+1}^{2} \\
& +\left(\frac{\bar{\ell}_{i, j}^{2}}{4}-\frac{1}{\tau_{i, j+1}}+1\right) \tilde{\gamma}_{i, j+1}^{2}+\frac{1}{4} \omega_{i, j}^{2}-\beta_{i, j} \widetilde{\varrho}_{i, j}^{T} \widehat{\varrho}_{i, j},
\end{aligned}
$$

where $\omega_{i, j}^{2}=\xi_{i, j}^{2}+\bar{B}_{i, j+1}^{2}$. Similar to (22), one has

$$
-\widetilde{\varrho}_{i, j}^{T} \widehat{\varrho}_{i, j} \leq-\frac{1}{2}\left\|\widetilde{\varrho}_{i, j}\right\|^{2}+\frac{1}{2}\left\|\varrho_{i, j}^{*}\right\|^{2},
$$

then we have

$$
\begin{aligned}
\dot{\Gamma}_{i, j}(t) \leq & -\left(\alpha_{i, j}-3\right) e_{i, j}^{2}-\left(\frac{1}{\tau_{i, j+1}}-\frac{\bar{\ell}_{i, j}^{2}}{4}-1\right) \tilde{\gamma}_{i, j+1}^{2}-\frac{\beta_{i, j}}{2}\left\|\widetilde{\varrho}_{i, j}\right\|^{2} \\
& +\left[\ell_{i, j} N\left(\kappa_{i, j}\right)+1\right] \dot{\kappa}_{i, j} \\
& +\frac{\beta_{i, j}}{2}\left\|\varrho_{i, j}^{*}\right\|^{2}+\frac{1}{4} \omega_{i, j}^{2}+\frac{1}{4} \ell_{i, j}^{2} e_{i, j+1}^{2} .
\end{aligned}
$$

Step $n_{i}$. At this final step, the actual controller $v_{i}(t)$ is designed. The derivative of the error surface $e_{i, n_{i}}=x_{i, n_{i}}-\bar{\gamma}_{i, n_{i}}$ is obtained as

$$
\dot{e}_{i, n_{i}}=h_{i, n_{i}}\left(\bar{x}_{i, n_{i}}\right)+\ell_{i, n_{i}}\left(\bar{x}_{i, n_{i}}\right)\left[c\left(v_{i}\right)+\eta\left(v_{i}\right)\right]+d_{i, n_{i}}-\dot{\bar{\gamma}}_{i, n_{i}} .
$$


Define

$\Psi_{i, n_{i}}\left(\zeta_{i, n_{i}}\right):=h_{i, n_{i}}\left(\bar{x}_{i, n_{i}}\right)-\dot{\bar{\gamma}}_{i, n_{i}}$, $\zeta_{i, n_{i}}=\left[\bar{x}_{i, n_{i}} \dot{\bar{\gamma}}_{i, n}\right]^{T} \in \mathbb{R}^{n_{i}+1}$. This unknown smooth function $\Psi_{i, n_{i}}\left(\zeta_{i, n_{i}}\right)$ can be approximated by an RBFNN as

$$
\begin{aligned}
& \Psi_{i, n_{i}}\left(\zeta_{i, n_{i}}\right)=\varrho_{i, n_{i}}^{* T} \vartheta_{i, n_{i}}+\varliminf_{i, n_{i}}
\end{aligned}
$$

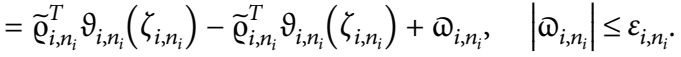

The actual controllers $v_{i}$ and adaptation laws $\kappa_{i, n_{i}}$ and $\widehat{\varrho}_{i, n_{i}}$ are designed as follows:

$$
\begin{aligned}
v_{i} & =N\left(\kappa_{i, n_{i}}\right)\left[\alpha_{i, n_{i}} e_{i, n_{i}}+\widehat{\varrho}_{i, n_{i}}^{T} \vartheta_{i, n_{i}}\right], \\
\dot{\kappa}_{i, n_{i}} & =\alpha_{i, n_{i}} e_{i, n_{i}}^{2}+e_{i, n_{i}} \widehat{\mathrm{\varrho}}_{i, n_{i}}^{T} \vartheta_{i, n_{i}}, \\
\dot{\mathrm{\varrho}}_{i, n_{i}} & =\Lambda_{i, n_{i}}\left[\vartheta_{i, n_{i}} e_{i, n_{i}}-\beta_{i, n_{i}} \widehat{\mathrm{\varrho}}_{i, n_{i}}\right],
\end{aligned}
$$

where $\Lambda_{i, n_{i}}$ is a positive-definite matrix, $\alpha_{i, n_{i}}>0$ and $\beta_{i, n_{i}}>0$ are the design parameters.

Applying the Lyapunov function $\Gamma_{i, n_{i}}(t)=(1 / 2) e_{i, n_{i}}^{2}+(1 / 2) \widetilde{\varrho}_{i, n_{i}}^{T} \Lambda_{i, n_{i}}^{-1} \widetilde{\varrho}_{i, n_{i}}$; similar to the aforementioned step, we have

$$
\begin{aligned}
\dot{\Gamma}_{i, n_{i}}(t)= & e_{i, n_{i}}\left[\widehat{\varrho}_{i, n_{i}}^{T} \vartheta_{i, n_{i}}\left(\zeta_{i, n_{i}}\right)-\widetilde{\varrho}_{i, n_{i}}^{T} \vartheta_{i, n_{i}}\left(\zeta_{i, n_{i}}\right)+{\varpi_{i, n_{i}}}\right. \\
& \left.+\ell_{i, n_{i}}\left[c\left(v_{i}\right)+\eta\left(v_{i}\right)\right]+d_{i, n_{i}}-\dot{\bar{\gamma}}_{i, n_{i}}\right] \\
\leq & -\left(\alpha i, n_{i}-1\right) e_{i, n_{i}}^{2}+\left[\ell_{i, n_{i}} c N\left(\kappa_{i, n_{i}}\right)+1\right] \dot{\kappa}_{i, n_{i}} \\
& -\frac{\beta_{i, n_{i}}}{2}\left\|\widetilde{\varrho}_{i, n_{i}}\right\|^{2}+\frac{\beta_{i, n_{i}}}{2}\left\|\varrho_{i, n_{i}}^{*}\right\|^{2}+\frac{1}{4} \omega_{i, n_{i}}^{2},
\end{aligned}
$$

where $\omega_{i, n_{i}}=\bar{\ell}_{i, n_{i}} \bar{\eta}+\bar{d}_{i, n_{i}}+\varepsilon_{i, n_{i}}$.

\section{Stability Analysis}

Theorem 1. Suppose that the initial conditions are bounded and the design parameters fulfill the following inequalities (44), then the output consensus problem for MAS (1) can be addressed by the controllers (10), (26), and (40) combining with adaptation laws (11), (12), (9), (28), (41), and (42), as well as the filters (18) and (32) under Assumptions 1-4. Moreover, all signals in the closed-loop MAS are SUUB, and the output consensus error can converge to a small region around the origin provided that the design parameters are appropriately selected:

$$
\left\{\begin{array}{l}
\alpha_{i, 1}>3, \\
\alpha_{i, j+1}>\frac{\bar{\ell}_{i, j}^{2}}{4}+3, \quad j=1,2, \ldots, n_{i}-2, \\
\alpha_{i, n_{i}}>1, \\
\frac{1}{\tau_{i, j+1}}>\frac{\bar{\ell}_{i, j}^{2}}{4}+1, \quad j=1,2, \ldots, n_{i}-1 .
\end{array}\right.
$$

Proof. Select the following constants $\mu_{i, 1}>0$ and $\nu_{i, 1}>0$ as

$$
\begin{aligned}
& \mu_{i, 1}=\min \left\{2\left(r_{i}+d_{i}\right)\left(\alpha_{i, 1}-3\right), \frac{\beta_{i, 1}}{\lambda_{\max }\left(\Lambda_{i, 1}^{-1}\right)}, 2\left(\frac{1}{\tau_{i, 2}}-\frac{\bar{\ell}_{i, 1}^{2}}{4}-1\right)\right\}, \\
& v_{i, 1}=\frac{\beta_{i, 1}\left\|\mathrm{e}_{i, j}^{*}\right\|^{2}}{2}+\frac{\omega_{i, 1}^{2}}{4} .
\end{aligned}
$$

From (23), one has

$$
\dot{\Gamma}_{i, 1}(t) \leq-\mu_{i, 1} \Gamma_{i, 1}(t)+\left[\ell_{i, 1} N\left(\kappa_{i, 1}\right)+1\right] \dot{\kappa}_{i, 1}+v_{i, 1}+\frac{1}{4} e_{i, 1}^{2} e_{i, 2}^{2} .
$$

Multiplying (46) by $e^{\mu_{i, 1} t}$ and then integrating it with respect to $t$ over $[0, t]$, one has

$$
\begin{aligned}
\Gamma_{i, 1}(t) \leq & \frac{\nu_{i, 1}}{\mu_{i, 1}}+\Gamma_{i, 1}(0)+e^{-\mu_{i, 1} 1} \int_{0}^{t}\left[\ell_{i, 1} N\left(\kappa_{i, 1}\right)+1\right] \dot{\kappa}_{i, 1} e^{\mu_{i, 1} s} \mathrm{~d} s \\
& +\frac{1}{4} e^{-\mu_{i, 1} t} \int_{0}^{t} \ell_{i, 1}^{2} e_{i, 2}^{2} e^{\mu_{i, 1} s} \mathrm{~d} s .
\end{aligned}
$$

we have

Since $e^{-\mu_{i, 1} t} \int_{0}^{t} \ell_{i, 1}^{2} e_{i, 2}^{2} e^{\mu_{i, 1} s} \mathrm{~d} s \leq\left(1 / \mu_{i, 1}\right) \bar{\ell}_{i, 1}^{2} \sup _{s \in[0, t]}\left[e_{i, 2}^{2}(s)\right]$,

$$
\Gamma_{i, 1}(t) \leq \rho_{i, 1}+e^{-\mu_{i, 1}} \int_{0}^{t}\left[\ell_{i, 1} N\left(\kappa_{i, 1}\right)+1\right] \dot{\kappa}_{i, 1} e^{\mu_{i, 1} s} \mathrm{~d} s,
$$

where

$\rho_{i, 1}=\left(v_{i, 1} / \mu_{i, 1}\right)+\Gamma_{i, 1}(0)+\left(1 / 4 \mu_{i, 1}\right) \bar{\ell}_{i, 1}^{2} \sup _{s \in[0, t]}\left[e_{i, 2}^{2}(s)\right]$.

Then, according to Lemma 1 , we can conclude that $\Gamma_{i, 1}(t)$, $e_{i, 1}(t), \kappa_{i, 1}(t), \widetilde{\varrho}_{i, 1}(t)$, and $\int_{0}^{t}\left[\ell_{i, 1} N\left(\kappa_{i, 1}\right)+1\right] \dot{\kappa}_{i, 1} \mathrm{~d} s$ are all SUUB on $[0, T)$. Let $\varsigma_{i, 1}>0$ be the upper bound of $\int_{0}^{t}\left[\ell_{i, 1} N\left(\kappa_{i, 1}\right)+1\right] \dot{\kappa}_{i, 1} \mathrm{~d} s$, i.e., $\left|\int_{0}^{t}\left[\ell_{i, 1} N\left(\kappa_{i, 1}\right)+1\right] \dot{\kappa}_{i, 1} \mathrm{~d} s\right| \leq \varsigma_{i, 1}$.

For $j=2, \ldots, n_{i}-1$, select the following constants $\mu_{i, j}>0$ and $v_{i, j}>0$ as

$$
\begin{aligned}
& \mu_{i, j}=\min \left\{2\left(\alpha_{i, j}-3\right), \frac{\beta_{i, j}}{\lambda_{\max }\left(\Lambda_{i, j}^{-1}\right)}, 2\left(\frac{1}{\tau_{i, j+1}}-\frac{\bar{\ell}_{i, j}^{2}}{4}-1\right)\right\}, \\
& v_{i, j}=\frac{\beta_{i, j}\left\|\varrho_{i, j}^{*}\right\|^{2}}{2}+\frac{\omega_{i, j}^{2}}{4} .
\end{aligned}
$$

From (37), we have

$$
\dot{\Gamma}_{i, j}(t) \leq-\mu_{i, j} \Gamma_{i, j}(t)+\left[\ell_{i, j} N\left(\kappa_{i, j}\right)+1\right] \dot{\kappa}_{i, j}+v_{i, j}+\frac{1}{4} \ell_{i, j}^{2} e_{i, j+1}^{2} .
$$

Similar to (48), one has

$$
\Gamma_{i, j}(t) \leq \rho_{i, j}+e^{-\mu_{i, j} t} \int_{0}^{t}\left[\ell_{i, j} N\left(\kappa_{i, j}\right)+1\right] \dot{\kappa}_{i, j} e^{\mu_{i, j} s} \mathrm{~d} s,
$$


where $\quad \rho_{i, j}=\left(v_{i, j} / \mu_{i, j}\right)+\Gamma_{i, j}(0)+\left(1 /\left(4 \mu_{i, j}\right)\right) \bar{\ell}_{i, j}^{2} \sup _{s \in[0, t]}$ $\left[e_{i, j+1}^{2}(s)\right]$. Then, according to Lemma 1 , one can conclude that $\Gamma_{i, j}(t), e_{i, j}(t), \kappa_{i, j}(t), \widetilde{\varrho}_{i, j}(t)$, and $\int_{0}^{t}\left[\ell_{i, j} N\left(\kappa_{i, j}\right)+1\right] \dot{\kappa}_{i, j} \mathrm{~d} s$ are all SUUB. Let $\varsigma_{i, j}>0$ be the upper bound of $\int_{0}^{t}\left[\ell_{i, j} N\left(\kappa_{i, j}\right)+1\right] \dot{\kappa}_{i, j} \mathrm{~d} s$, i.e., $\left|\int_{0}^{t}\left[\ell_{i, j} N\left(\kappa_{i, j}\right)+1\right] \dot{\kappa}_{i, j} \mathrm{~d} s\right| \leq \varsigma_{i, j}$.

Finally, applying the Lyapunov function,

$$
\begin{aligned}
\Gamma(t)= & \sum_{i=1}^{N} \sum_{j=1}^{n_{i}} \Gamma_{i, j}(t)=\sum_{i=1}^{N}\left[\frac{1}{2\left(r_{i}+d_{i}\right)} e_{i, 1}^{2}+\frac{1}{2} \sum_{j=2}^{n_{i}} e_{i, j}^{2}\right. \\
& \left.+\frac{1}{2} \sum_{j=1}^{n_{i}-1} \widetilde{\gamma}_{i, j+1}^{2}+\frac{1}{2} \sum_{j=1}^{n_{i}} \widetilde{\mathrm{Q}}_{i, j}^{T} \Lambda_{i, j}^{-1} \widetilde{\mathrm{Q}}_{i, j}\right] .
\end{aligned}
$$

Denote

$$
\begin{aligned}
0<\delta \leq & \min _{\substack{i=1,2, \ldots, N \\
j=1,2, \ldots, n_{i}-1}}\left\{\mu_{i, j}, 2\left(\alpha_{i, j+1}-3-\frac{\bar{\ell}_{i, j}^{2}}{4}\right), 2\left(\alpha_{i, n_{i}}-1\right)\right. \\
& \left.\frac{\beta_{i, n_{i}}}{\lambda_{\max }\left(\Lambda_{i, n_{i}}^{-1}\right)}\right\}, \\
\chi= & \sum_{i=1}^{N} \sum_{j=1}^{n_{i}} \frac{\beta_{i, j}}{2}\left\|\mathrm{\varrho}_{i, j}^{*}\right\|^{2}+\frac{1}{4} \sum_{i=1}^{N} \sum_{j=1}^{n_{i}} \omega_{i, j}^{2}, \\
\hat{\ell}_{i, j}= & \begin{cases}\ell_{i, j}, & j=1,2, \ldots, n_{i}-1, \\
c \ell_{i, n_{i},} & j=n_{i},\end{cases}
\end{aligned}
$$

then substitute (23), (37), and (43) into the time derivative of (52), and based on (44), we have

$$
\begin{aligned}
\dot{\Gamma}(t)= & -\sum_{i=1}^{N}\left(\alpha_{i, 1}-3\right) e_{i, 1}^{2}-\sum_{i=1}^{N} \sum_{j=2}^{n_{i}-1}\left(\alpha_{i, j}-3-\frac{\bar{\ell}_{i, j-1}^{2}}{4}\right) e_{i, j}^{2}-\sum_{i=1}^{N}\left(\alpha_{i, n_{i}}-1\right) e_{i, n_{i}}^{2} \\
& -\sum_{i=1}^{N} \sum_{j=1}^{n_{i}-1}\left(\frac{1}{\tau_{i, j+1}}-\sum_{i=1}^{N} \frac{\bar{\ell}_{i, j}^{2}}{4}-1\right) \widetilde{\gamma}_{i, j+1}^{2}-\sum_{i=1}^{N} \sum_{j=1}^{n_{i}} \frac{\beta_{i, j}}{2}\left\|\widetilde{\varrho}_{i, j}\right\|^{2} \\
& +\sum_{i=1}^{N} \sum_{j=1}^{n_{i}}\left[\widehat{\ell}_{i, j} N\left(\kappa_{i, j}\right)+1\right] \dot{\kappa}_{i, j}+\sum_{i=1}^{N} \sum_{j=1}^{n_{i}} \frac{\beta_{i, j}}{2}\left\|\varrho_{i, j}^{*}\right\|^{2}+\frac{1}{4} \sum_{i=1}^{N} \sum_{j=1}^{n_{i}} \omega_{i, j}^{2} \\
\leq & -\delta \Gamma(t)+\sum_{i=1}^{N} \sum_{j=1}^{n_{i}}\left[\hat{\ell}_{i, j} N\left(\kappa_{i, j}\right)+1\right] \dot{\kappa}_{i, j}+\chi .
\end{aligned}
$$

Multiplying the above inequality by $e^{\delta t}$ and then integrating it with respect to $t$ over $[0, t]$, we have

$$
\begin{aligned}
\Gamma(t) \leq & \frac{\chi}{\delta}+e^{-\delta t}\left[\Gamma(0)-\frac{\chi}{\delta}\right]+e^{-\delta t} \sum_{i=1}^{N} \sum_{j=1}^{n_{i}} \int_{0}^{t}\left[\hat{\ell}_{i, j} N\left(\kappa_{i, j}\right)+1\right] \\
& \times \dot{\kappa}_{i, j} e^{\delta s} \mathrm{~d} s, \\
\leq & \frac{\chi}{\delta}+\Gamma(0)+\sum_{i=1}^{N} \sum_{j=1}^{n_{i}} \int_{0}^{t}\left[\hat{\ell}_{i, j} N\left(\kappa_{i, j}\right)+1\right] \dot{\kappa}_{i, j} e^{-\delta(t-s)} \mathrm{d} s, \\
\leq & \frac{\chi}{\delta}+\Gamma(0)+\sum_{i=1}^{N} \sum_{j=1}^{n_{i}-1} \varsigma_{i, j}+e^{-\delta t} \sum_{i=1}^{N} \int_{0}^{t}\left[\hat{\ell}_{i, n_{i}} N\left(\kappa_{i, n_{i}}\right)+1\right] \\
& \times \dot{\kappa}_{i, n_{i}} e^{\delta s} \mathrm{~d} s .
\end{aligned}
$$

By Lemma 1, it can be concluded that $\Gamma(t), e_{i, n_{i}}(t)$, $\kappa_{i, n_{i}}(t), \quad \widetilde{\varrho}_{i, n_{i}}(t)$, and $\sum_{i=1}^{N} \int_{0}^{t}\left[\hat{\ell}_{i, n_{i}} N\left(\kappa_{i, n_{i}}\right)+1\right] \dot{\kappa}_{i, n_{i}} \mathrm{~d} s$ are all
SUUB; i.e., all the signals in the closed-loop system are SUUB. Besides, from (52) and (55), one has

$$
\begin{aligned}
\sum_{i=1}^{N} \frac{1}{2\left(r_{i}+d_{i}\right)} e_{i, 1}^{2} \leq & \Gamma(t) \leq \frac{\chi}{\delta}+e^{-\delta t}\left[\Gamma(0)-\frac{\chi}{\delta}\right] \\
& +e^{-\delta t} \sum_{i=1}^{N}\left[\hat{\ell}_{i, j} N\left(\kappa_{i, j}\right)+1\right] \dot{\kappa}_{i, j} e^{\delta s} \mathrm{~d} s .
\end{aligned}
$$

Since the matrix $\mathscr{L}+\mathscr{R}$ is nonsingular under Assumption 4, denote its minimum singular value as $\underline{\sigma}$, which is a positive constant [10]. Define $M=\max _{i}\left\{r_{i}+d_{i}\right\}$ and $z_{1}=\left[z_{1,1}, z_{2,1}\right.$, $\left.\ldots, z_{N, 1}\right]^{T}=(\mathscr{L}+\mathscr{R})\left(y-y_{0} 1\right)$ with $y=\left[y_{1}, y_{2}, \ldots, y_{N}\right]^{T}$ and $1=[1,1, \ldots, 1] T \in \mathbb{R}^{N}$. Then, we have

$$
\lim _{t \rightarrow \infty}\left\|y-y_{0} 1\right\| \leq \sqrt{\frac{2 M \chi}{\underline{\sigma} \delta}} .
$$

Hence, the output consensus error can converge to a small region around the origin provided that the design parameters $\alpha_{i, j}, \beta_{i, j}$, and $\tau_{i, j+1}$ are appropriately selected. 


\section{Numerical Simulation}

To test the availability of the proposed control algorithm, consider the following uncertain MAS with 3 followers:

$$
\left\{\begin{array}{l}
\dot{x}_{i 1}=h_{i 1}\left(x_{i 1}\right)+\ell_{i 1}\left(x_{i 1}\right) x_{i 2}+d_{i 1}(t), \\
\dot{x}_{i 2}=h_{i 2}\left(\bar{x}_{i 2}\right)+\ell_{i 2}\left(\bar{x}_{i 2}\right) \sigma\left(v_{i}\right)+d_{i 2}(t), \\
y_{i}=x_{i 1}, \\
i=1,2,3
\end{array}\right.
$$

where $h_{11}\left(x_{11}\right)=-2 x_{11}+x_{11}^{2}, \ell_{11}\left(x_{11}\right)=-\left[3+2 \sin \left(x_{11}^{2}\right)\right]$, $h_{12}\left(\bar{x}_{12}\right)=x_{11} x_{12}^{2}, \quad \ell_{12}\left(\bar{x}_{12}\right)=-\left[4+2 \cos \left(x_{11} x_{12}^{2}\right)\right]$, $h_{21}\left(x_{21}\right)=-x_{21}+\sin \left(x_{21}\right), \quad \ell_{21}\left(x_{21}\right)=5-3 \cos \left(x_{21}\right)$, $h_{22}\left(\bar{x}_{22}\right)=-\cos \left(x_{21}\right) x_{22}, \quad \ell_{22}\left(\bar{x}_{22}\right)=-\left[2-\sin \left(x_{21} x_{22}^{3}\right)\right]$, $h_{31}\left(x_{31}\right)=-x_{31} \cos \left(x_{31}^{2}\right), \quad \ell_{31}\left(x_{31}\right)=2+\sin \left(x_{31}^{2}\right)$, $h_{32}\left(\bar{x}_{32}\right)=-0.5 x_{31} x_{32}$, and $\ell_{32}\left(\bar{x}_{32}\right)=-\left[2+\cos \left(x_{31} x_{32}\right)\right]$ are the unknown continuous functions and $d_{11}(t)=d_{21}(t)=-0.01 \sin (t)$,

$d_{12}(t)=d_{22}(t)=0.02 \cos (t), \quad d_{31}(t)=-0.02 \cos (t), \quad$ and $d_{32}(t)=0.01 \sin (t)$ are the unknown disturbances. The leader's output is given by $y_{0}(t)=0.4 \sin (t)$. It is easy to verify that the conditions in Assumptions 1-3 are all satisfied. Figure 1 depicts the communication digraph of the leader and the 3 followers.

In simulation, RBFNNs $\varrho_{i, 1} T \vartheta_{i, 1}\left(\zeta_{i, 1}\right)$ and $\varrho_{i, 2} T \vartheta_{i, 2}\left(\zeta_{i, 2}\right)$, which contain 11 neurons with centers spaced evenly in $[-1,1]$ and widths being equal to $w_{1}=0.5$ and $w_{2}=5$, respectively, are used to approximate the unknown continuous functions $h_{i 1}\left(x_{i 1}\right)$ and $h_{i 2}\left(\bar{x}_{i 2}\right), i=1,2,3$. The initial conditions are chosen as

$$
\begin{aligned}
{\left[x_{11}\right.} & \left.(0), x_{12}(0), x_{21}(0), x_{22}(0), x_{31}(0), x_{32}(0)\right]^{T} \\
& =[0.3,0.01,0.2,0.01,0.1,0.01]^{T}, \\
v^{*} & =5 \\
\alpha_{i, 1} & =20 \\
\alpha_{i, 2} & =10 \\
\Lambda_{i, j} & =0.1 I \\
\tau_{i, j} & =0.01, \\
\beta_{i, j} & =0.01, \\
\kappa_{i, j}(0) & =0, \\
\widehat{\varrho}_{i, j}(0) & =0, \\
i & =1,2,3 ; j=1,2 .
\end{aligned}
$$

Figures 2-4 display the evolution of the output trajectories of the 3 followers and the leader, the corresponding output consensus errors, and the actual controllers $v_{i}$, respectively. One can see that the outputs of the 3 followers can track the leader's output since the output consensus error converges to a small neighborhood of zero. Additionally, it can be seen that there exist large differences between $v_{i}$ and the saturation level constant $v^{*}$, which indicates the significant saturation effect. The simulation results illustrate that the proposed control algorithm is effective.

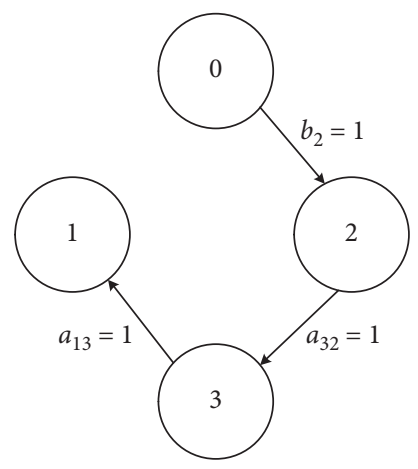

Figure 1: Communication graph, where 0 denotes the leader and 1-3 denote the 3 followers, respectively.

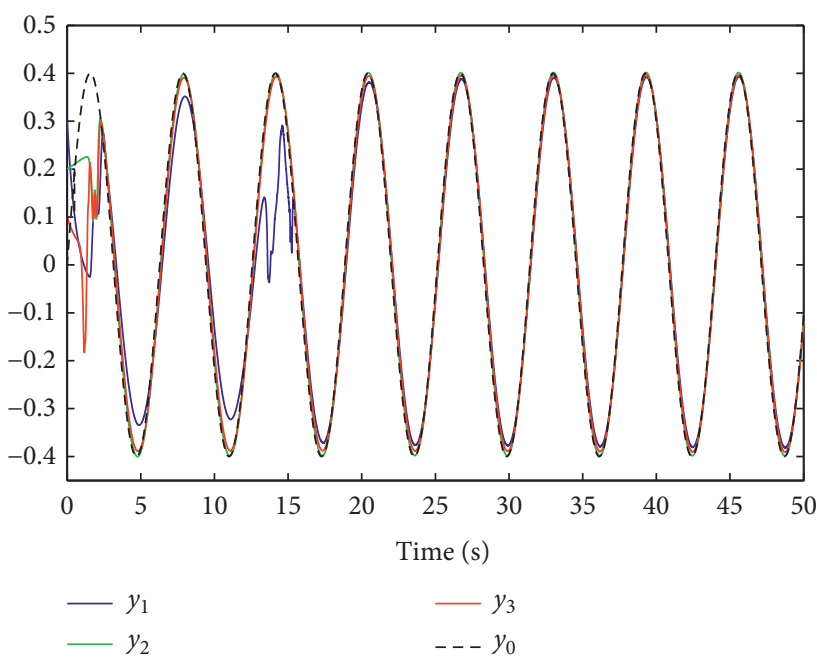

FIGURE 2: Evolution of the output trajectories.

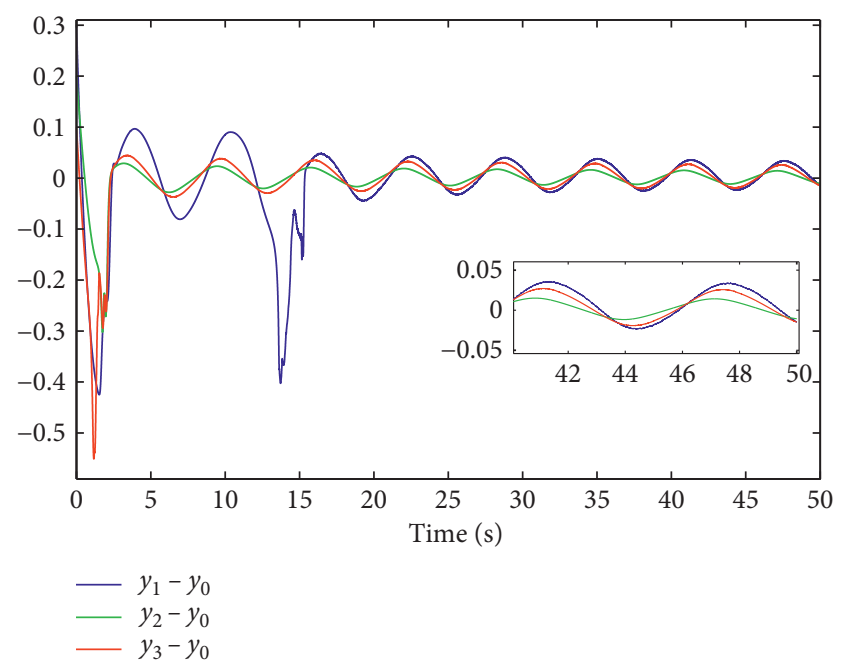

FIGURE 3: Evolution of the output consensus errors.

Remark 3. The reason that why there exist large fluctuations in the output trajectories of $y_{i}$ lies in the large fluctuations of the actual controllers $v_{i}$ (see Figure 4 ). Because the Nussbaum gain function $N\left(\kappa_{i, 2}\right)=\kappa_{i, 2}^{2} \cos \left((\pi / 2) \kappa_{i, 2}\right)$ will be very 

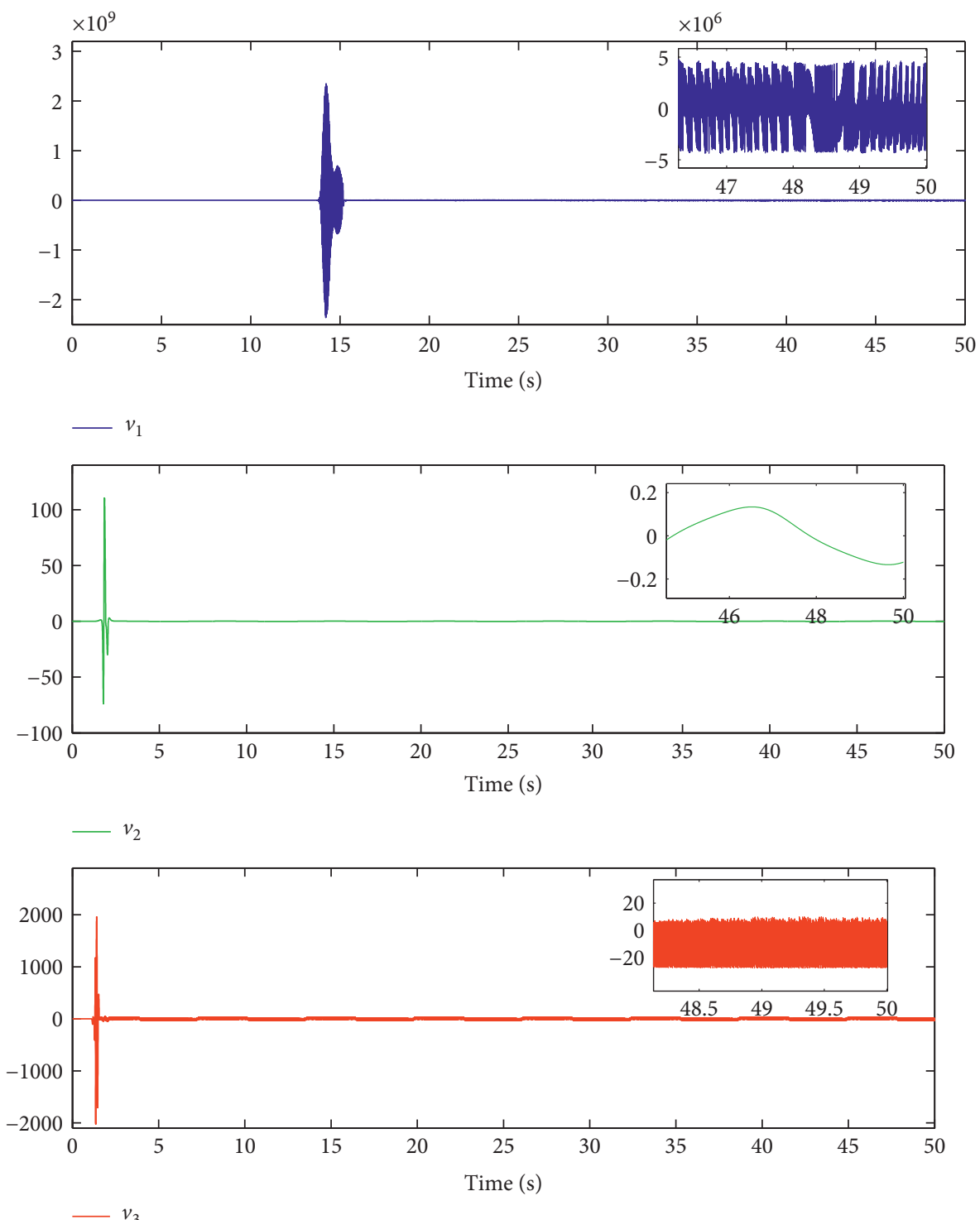

FIgURE 4: Evolution of the actual controllers $v_{i}$.

large (positively or negatively) if $\kappa_{i, 2}$ becomes very large, correspondingly the actual controllers $v_{i}$ will be very large according to equation (40). To reduce the values of $\kappa_{i, 2}$, the design parameters $\alpha_{i, 2}$ should be turned smaller. However, from the proof of Theorem 1, we know that smaller $\alpha_{i, 2}$ may lead to a smaller value of $\delta$ and thereby causes a larger output consensus error (see equation (59)). In a word, there is a tradeoff between smaller output consensus errors and smooth output trajectories. This is another problem worthy to explore, which may be our future work.

\section{Conclusion}

In this paper, an output consensus scheme for a class of highorder affine nonlinear MAS with unknown control gains and input saturation under a directed communication graph is proposed. Combining the adaptive RBFNN approximation and the DSC methodology, consensus control laws are developed to guarantee the semiglobal uniform ultimate boundedness for all signals in the closed-loop system. Moreover, the output consensus error can converge to a small region around the origin provided that the design parameters are appropriately selected. The effectiveness of the proposed control algorithm is revealed by a numerical simulation.

\section{Data Availability}

No data were used to support this study.

\section{Conflicts of Interest}

The authors declare that they have no conflicts of interest.

\section{Acknowledgments}

This research was partially supported by the National Natural Science Foundation of China (Grant nos. 61703175 
and 11626111), the Natural Science Foundation of Huizhou University (Grant no. hzu201806), the Natural Science Foundation of Jiangsu Province, China (Grant no. BK20171019), and the Research Fund for the Doctoral Program of Huizhou University (Grant no. 2015JB020).

\section{References}

[1] H. Zhang, Z. Chen, and M. Fan, "Collaborative control of multivehicle systems in diverse motion patterns," IEEE Transactions on Control Systems Technology, vol. 24, no. 4, pp. 1488-1494, 2016.

[2] T. Han, M. Chi, Z.-H. Guan, B. Hu, J.-W. Xiao, and Y. Huang, "Distributed three-dimensional formation containment control of multiple unmanned aerial vehicle systems," Asian Journal of Control, vol. 19, no. 3, pp. 1103-1113, 2017.

[3] B. Liu, H. Zhang, H. Meng, D. Fu, and H. Su, "Scanning-chain formation control for multiple unmanned surface vessels to pass through water channels," IEEE Transactions on Cybernetics, 2020.

[4] Y. Zhang and Y.-P. Tian, "A fully distributed weight design approach to consensus kalman filtering for sensor networks," Automatica, vol. 104, pp. 34-40, 2019.

[5] G. Wen, X. Yu, Z. W. Liu, and W. Yu, "Adaptive consensusbased robust strategy for economic dispatch of smart grids subject to communication uncertainties," IEEE Transactions on Industrial Informatics, vol. 14, no. 6, pp. 2484-2496, 2018.

[6] H. Zhang, M. Fan, Y. Wu et al., "Ultrafast synchronization via local observation," New Journal of Physics, vol. 21, no. 1, Article ID 013040, 2019.

[7] D. Chen, M. Kang, and W. Yu, "Probabilistic causal inference for coordinated movement of pigeon flocks," $E P L$, vol. 130, p. 28004, 2020.

[8] D. Chen, W. Li, X. Li, W. Yu, and Y. Sun, "Effects of measurement noise on flocking dynamics of cucker-smale sytems," IEEE Transactions on Circuits and Systems II: Express Briefs, 2019.

[9] X. Xu, L. Liu, and G. Feng, "Consensus of single integrator multi-agent systems with directed topology and communication delays," Control Theory and Technology, vol. 14, no. 1, pp. 21-27, 2016.

[10] W. Ren, "On consensus algorithms for double-integrator dynamics," IEEE Transactions on Automatic Control, vol. 53, no. 6, pp. 1503-1509, 2008.

[11] G. Wen, H.-T. Zhang, W. Yu, Z. Zuo, and Y. Zhao, "Coordination tracking of multi-agent dynamical systems with general linear node dynamics," International Journal of Robust and Nonlinear Control, vol. 27, no. 9, pp. 1526-1546, 2017.

[12] X. Wang, H. Su, M. Z. Q. Chen, X. F. Wang, and G. Chen, "Reaching non-negative edge consensus of networked dynamical systems," IEEE Transactions on Cybernetics, vol. 48, no. 9, pp. 2712-2722, 2018.

[13] H. Ji, H. Zhang, Z. Ye, H. Zhang, B. Xu, and G. Chen, "Stochastic consensus control of second-order nonlinear multiagent systems with external disturbances," IEEE Transactions on Control of Network Systems, vol. 5, no. 4, pp. 1585-1596, 2018.

[14] M. Fan, Z. Chen, and H.-T. Zhang, "Semi-global consensus of nonlinear second-order multi-agent systems with measurement output feedback," IEEE Transactions on Automatic Control, vol. 59, no. 8, pp. 2222-2227, 2014.
[15] W. Qin, Z.-X. Liu, and Z. Chen, "Observer-based consensus for nonlinear multi-agent systems with intermittent communication," Neurocomputing, vol. 154, pp. 230-238, 2015.

[16] H. Meng, Z. Chen, L. Zhu, and R. Middleton, "Consensus of a class of second-order nonlinear heterogeneous multi-agent systems with uncertainty and communication delay," International Journal of Robust and Nonlinear Control, vol. 26, no. 15, pp. 3311-3329, 2016.

[17] X. Yin, H. Su, and D. Yue, "Consensus of fractional-order heterogeneous multi-agent systems," IET Control Theory \& Applications, vol. 7, no. 2, pp. 314-322, 2013.

[18] L. Chen, Y. W. Wang, W. Yang, and J. W. Xiao, "Robust consensus of fractional-order multi-agent systems with input saturation and external disturbances," Neurocomputing, vol. 303, pp. 11-19, 2018.

[19] Y. Xie and Z. Lin, "Global optimal consensus for higher-order multi-agent systems with bounded controls," Automatica, vol. 99, pp. 301-307, 2019.

[20] C. C. Hua, K. Li, and X. P. Guan, "Leader-following output consensus for high-order nonlinear multiagent systems," IEEE Transactions on Automatic Control, vol. 64, no. 3, pp. 1156-1161, 2019.

[21] J. Peng and X. Ye, "Cooperative control of multiple heterogeneous agents with unknown high-frequency-gain signs," Systems \& Control Letters, vol. 68, pp. 51-56, 2014.

[22] M. Radenkovic and M. Tadi, "Multi-agent adaptive consensus of networked systems on directed graphs," International Journal of Adaptive Control and Signal Processing, vol. 30, no. 1, pp. 46-59, 2016.

[23] W. Chen, X. Li, W. Ren, and C. Chen, "Adaptive consensus of multi-agent systems with unknown identical control directions based on a novel nussbaum-type function," IEEE Transactions on Automatic Control, vol. 59, no. 7, pp. 18871892, 2014.

[24] Q. Ma, "Cooperative control of multi-agent systems with unknown control directions," Applied Mathematics and Computation, vol. 292, pp. 240-252, 2017.

[25] J. Huang, Y. Song, W. Wang, C. Wen, and G. Li, "Fully distributed adaptive consensus control of a class of high-order nonlinear systems with a directed topology and unknown control directions," IEEE Transactions on Cybernetics, vol. 48, no. 8, pp. 2349-2356, 2018.

[26] C. Chen, C. Wen, Z. Liu, K. Xie, Y. Zhang, and C. P. Chen, "Adaptive consensus of nonlinear multi-agent systems with non-identical partially unknown control directions and bounded modelling errors," IEEE Transactions on Automatic Control, vol. 62, no. 9, pp. 4654-4659, 2016.

[27] M. Fan, H.-T. Zhang, and Z. Lin, "Distributed semiglobal consensus with relative output feedback and input saturation under directed switching networks," IEEE Transactions on Circuits and Systems II: Express Briefs, vol. 62, no. 8, pp. 796-800, 2015.

[28] M. Fan and M. Wang, "Second-order consensus for a class of uncertain multi-agent systems subject to input saturation," Transactions of the Institute of Measurement and Control, vol. 41, no. 7, pp. 1957-1964, 2019.

[29] X. Wang, H. Su, M. Z. Q. Chen, and X. F. Wang, "Observerbased robust coordinated control of multiagent systems with input saturation," IEEE Transactions on Neural Networks and Learning Systems, vol. 29, no. 5, pp. 1933-1946, 2018.

[30] X. Liu, J.-W. Xiao, D. Chen, and Y.-W. Wang, "Dynamic consensus of nonlinear time-delay multi-agent systems with input saturation: an impulsive control algorithm," Nonlinear Dynamics, vol. 97, no. 2, pp. 1699-1710, 2019. 
[31] D. Chen, T. Lu, X. Liu, and W. Yu, "Finite-time consensus of multiagent systems with input saturation and disturbance," International Journal of Robust and Nonlinear Control, 2020.

[32] H. Zhang and F. L. Lewis, "Adaptive cooperative tracking control of higher-order nonlinear systems with unknown dynamics," Automatica, vol. 48, no. 7, pp. 1432-1439, 2012.

[33] G. Wang, "Distributed control of higher-order nonlinear multi-agent systems with unknown non-identical control directions under general directed graphs," Automatica, vol. 110, Article ID 108559, 2019.

[34] M. Shahriari-kahkeshi and M. Taj, "Distributed adaptive consensus tracking control for uncertain non-linear multiagent systems with input saturation," IET Control Theory \& Applications, vol. 14, no. 13, pp. 2153-2162, 2019.

[35] W. Wang, D. Wang, Z. Peng, and H. Wang, "Cooperative adaptive fuzzy output feedback control for synchronization of nonlinear multi-agent systems in the presence of input saturation," Asian Journal of Control, vol. 18, no. 2, pp. 619-630, 2016.

[36] M. Fan and Y. Wu, "Global leader-following consensus of nonlinear multi-agent systems with unknown control directions and unknown external disturbances," Applied Mathematics and Computation, vol. 331, pp. 274-286, 2018.

[37] M. Krstic, I. Kanellakopoulos, and P. Kokotovic, Nonlinear and Adaptive Control Design, Wiley-Interscience, Hoboken, NJ, USA, 1995.

[38] Z. Zheng, C. Jin, M. Zhu, and K. Sun, "Trajectory tracking control for a marine surface vessel with asymmetric saturation actuators," Robotics and Autonomous Systems, vol. 97, pp. 83-91, 2017.

[39] X. Ye and J. Jiang, "Adaptive nonlinear design without a priori knowledge of control directions," IEEE Transactions on Automatic Control, vol. 43, no. 11, pp. 1617-1621, 1998.

[40] S. S. Ge, F. Hong, and T. H. Lee, "Adaptive neural control of nonlinear time-delay systems with unknown virtual control coefficients," IEEE Transactions on Systems, Man and Cybernetics, Part B (Cybernetics), vol. 34, no. 1, pp. 499-516, 2004.

[41] J. J. E. Slotine and W. Li, Applied Nonlinear Control, PrenticeHall, Upper Saddle River, NJ, USA, 1991.

[42] D. Wang and J. Huang, "Neural network-based adaptive dynamic surface control for a class of uncertain nonlinear systems in strict-feedback form," IEEE Transactions on Neural Networks, vol. 16, no. 1, pp. 195-202, 2005. 